\title{
Pathogen emergence in changing ecosystems: RAD-seq reveals long evolutionary history of Phellinus noxius in east Asia, Australia, and the Pacific Islands
}

\author{
Olga Kozhar ${ }^{1}$, Mee-Sook Kim² ${ }^{2}$ Jorge Ibarra Caballero ${ }^{3}$, Ned Klopfenstein ${ }^{4}$, Phil Cannon ${ }^{5}$, \\ and Jane Stewart ${ }^{3}$ \\ ${ }^{1}$ Affiliation not available \\ ${ }^{2}$ United States Department of Agriculture Forest Service Pacific Northwest Region \\ ${ }^{3}$ Colorado State University \\ ${ }^{4}$ United States Department of Agriculture Forest Service Rocky Mountain Research Station \\ ${ }^{5}$ United States Department of Agriculture Forest Service Forest Health Protection
}

July 19, 2021

\begin{abstract}
Emerging pathogens have been increasing exponentially over the last century. The knowledge on whether these organisms are native to ecosystems or have been recently introduced is often of great importance. Understanding the ecological and evolutionary processes promoting emergence can help to control their spread and forecast epidemics. Using restriction site-associated DNA sequencing data, we studied genetic relationships, pathways of spread, and evolutionary history of Phellinus noxius, an emerging root-rotting fungus of unknown origin, in eastern Asia, Australia, and the Pacific Islands. We analyzed patterns of genetic variation using Bayesian inference, maximum likelihood phylogeny, populations splits and mixtures measuring correlations in allele frequencies and genetic drift, and finally applied coalescent based theory using approximate Bayesian computation (ABC) with supervised machine learning. Population structure analyses revealed five genetic groups with signatures of complex recent and ancient migration histories. The most probable scenario of ancient pathogen spread is movement from west to east: from Malaysia to the Pacific Islands, with subsequent spread to Taiwan and Australia. Furthermore, ABC analyses indicate that $P$. noxius spread occurred thousands of generations ago, contradicting previous assumptions that it was recently introduced in multiple areas. Our results suggest that recent emergence of $P$. noxius in east Asia, Australia, and the Pacific Islands is likely driven by anthropogenic and natural disturbances, including deforestation, land-use change, severe weather events, and introduction of exotic plants. This study provides a novel example of utilization of genome wide allele frequency data to unravel dynamics of pathogen emergence under conditions of changing ecosystems.
\end{abstract}

\section{Hosted file}

Phellinus_main_text_7_7_2021.docx available at https://authorea.com/users/426415/articles/ 530943-pathogen-emergence-in-changing-ecosystems-rad-seq-reveals-long-evolutionaryhistory-of-phellinus-noxius-in-east-asia-australia-and-the-pacific-islands 
a

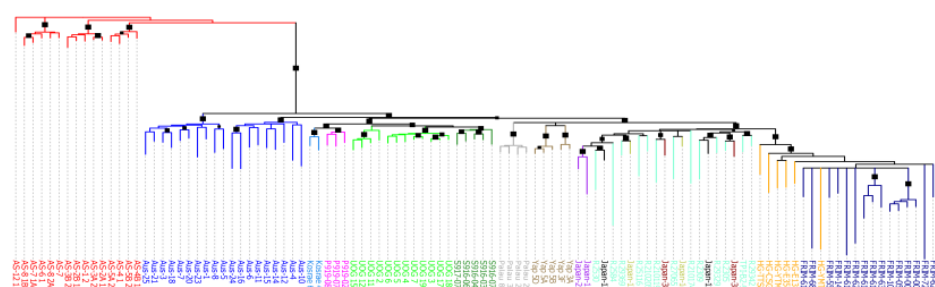

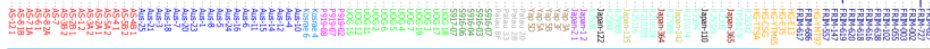

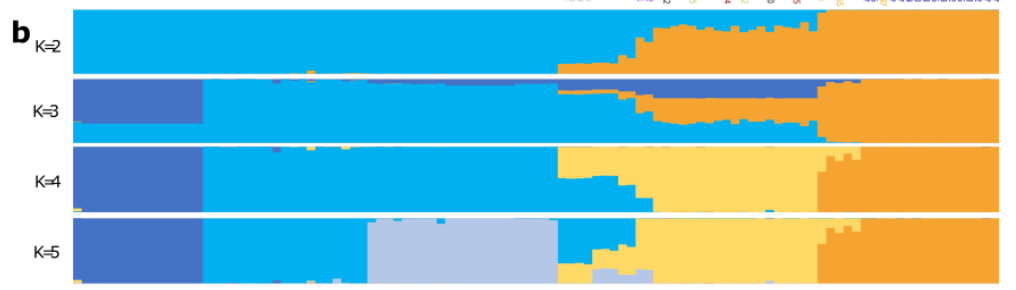

C

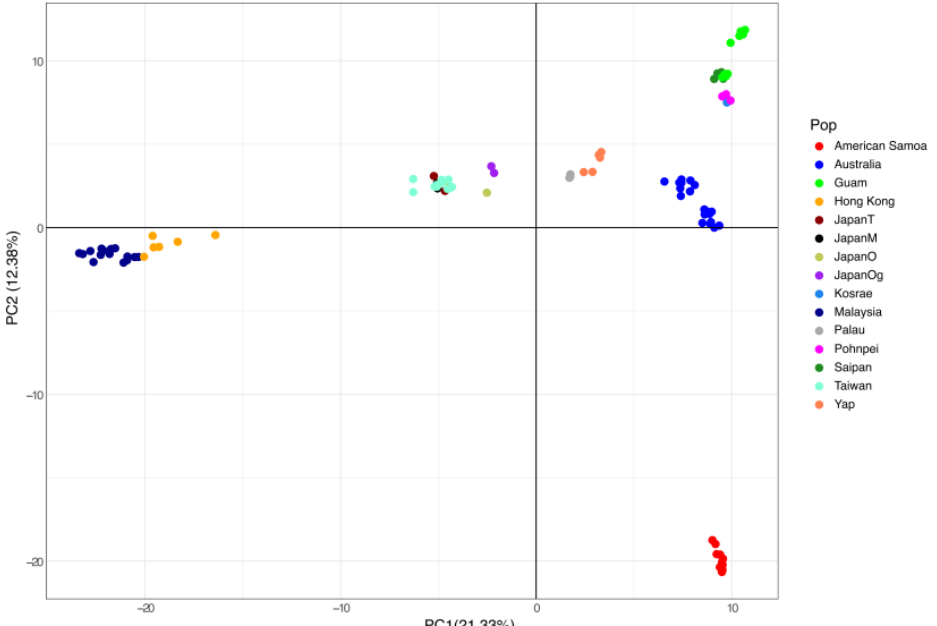

Fig. 1. Maximum likelihood phylogenetic tree (a), Structure Bayesian clustering (b), and Principa component analysis (PCA) of Phellinus noxius populations from 15 locations (c). (a) The phylogenetic tree was constructed with IQ-TREE v. 20.3. Different colors represent samples from different locations reflected in PCA legend (c). The branches with $\geq 95 \%$ likelihood support are indicated with black squares. The branch support was calculated with 1000 ultrafast bootstrap replicates. (b) Structure results $K$ from 2 to 5 , each support was calculate with 1000 ultrach bar represents posterior probability of sample assignment to color bar represents posterior probability of sample assignment to color coded genetic groups. (c) PCA represents distinct genetic groups within the studied dataset. Each dot indicates One individual. Percentages between brackets represent variance Adegenet v.2.1.3. For isolates and locations abbreviations see Table S1. 

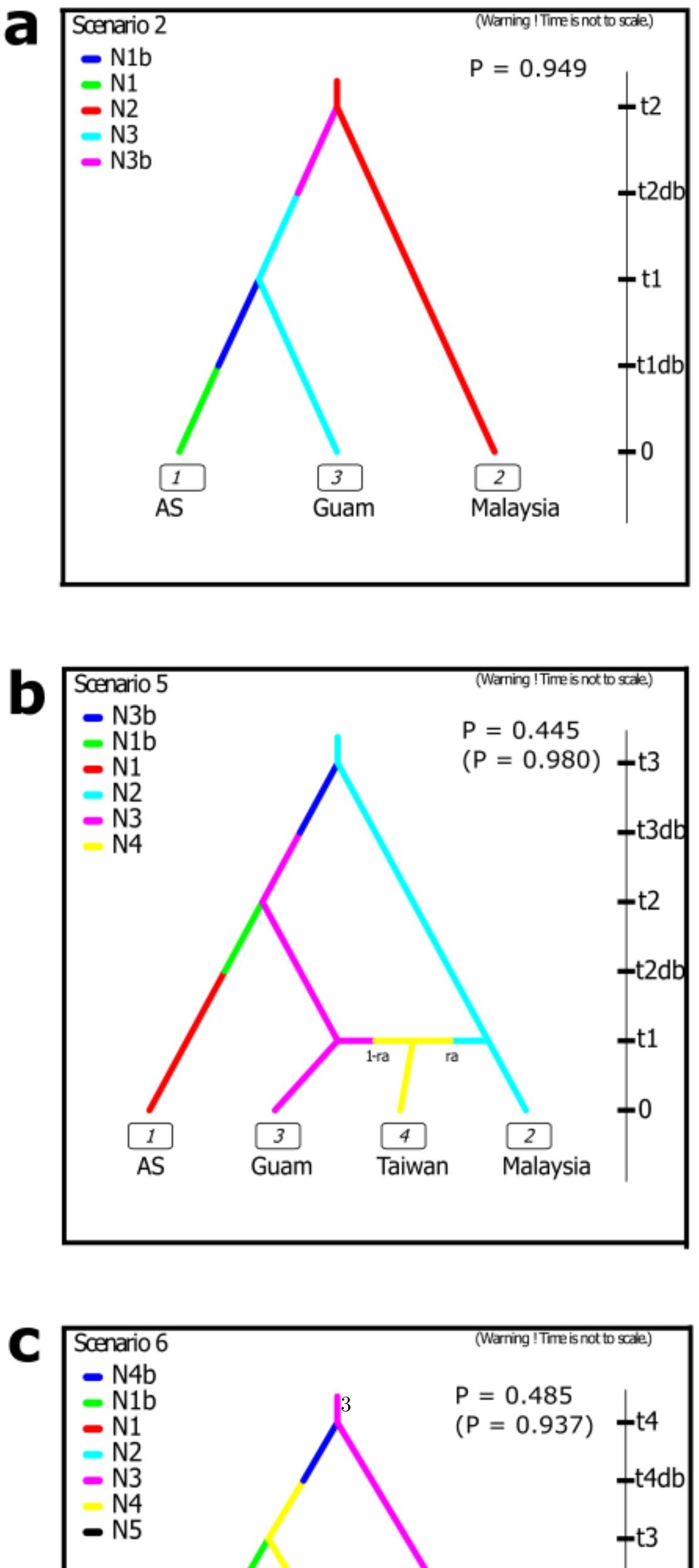
a

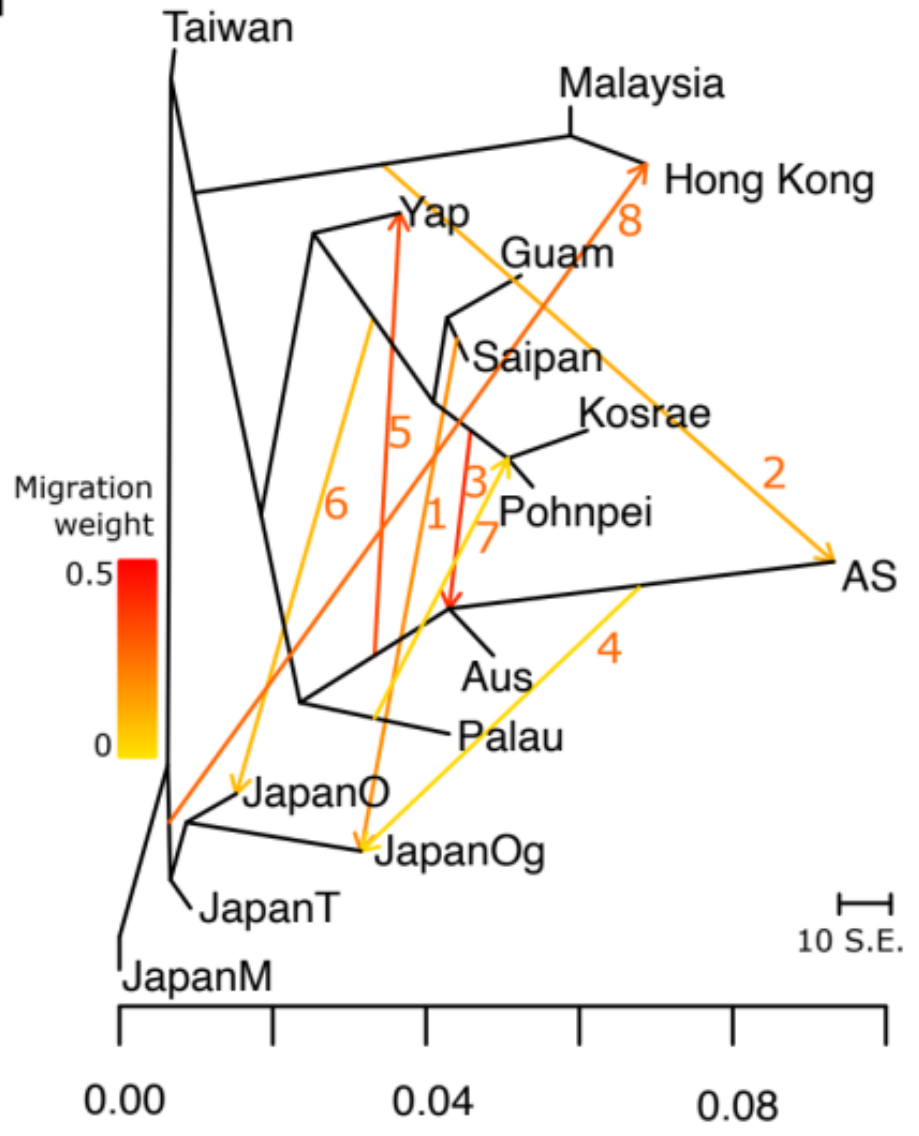

Drift parameter

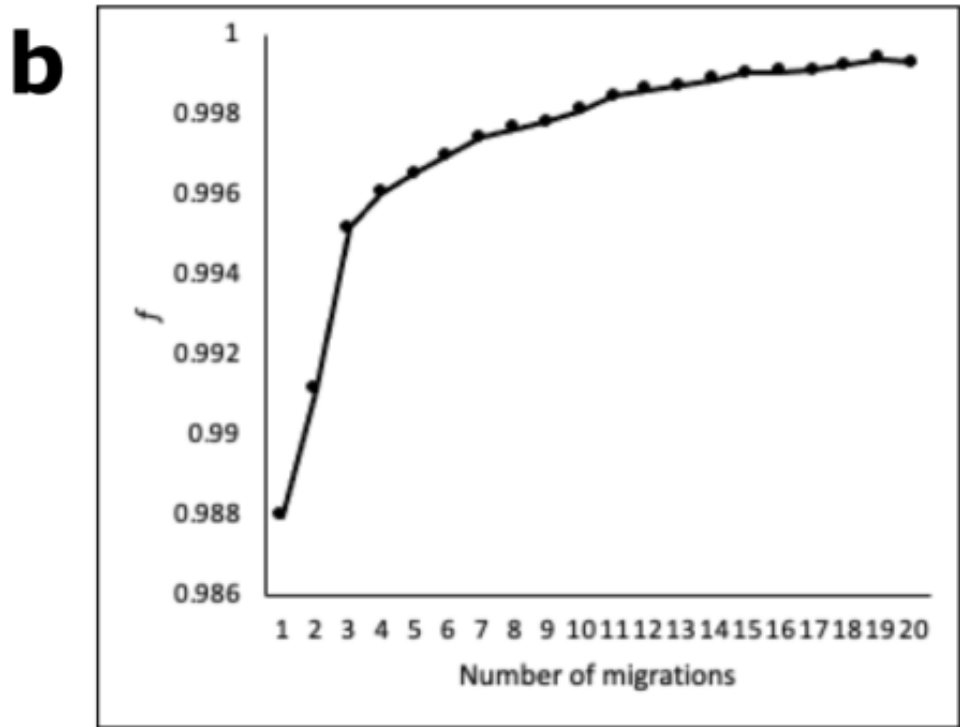

Fig. 3. (a) Phylogenetic network inferred by Treemix of the relationships among Phellinus noxius populations with eight migration edges that explain $99.8 \%$ variance in the model. The migration edges are numbered from 1 to 8 reflecting the order in 
a

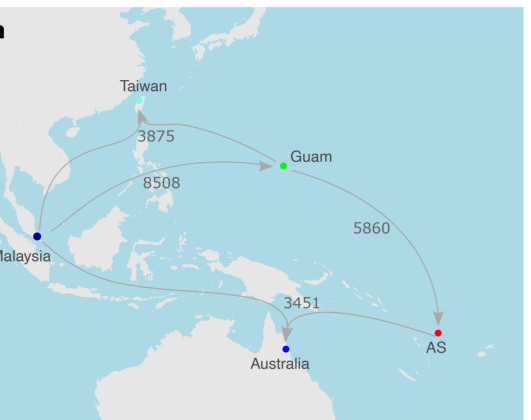

Fig. 4. (a) Invasion routes of Phellinus noxius determined with DIYABC Random Forest analyses. The analysis was performed among populations representing five genetic groups
identified in this study. Numbers next to the lines represent the order of invasion events. (b) Migration among Phellinus noxius populations sampled from 15 locations detected with Treemix. The width of the lines reflects the percentage of ancestry (migration weight) in recipient from donor population (see Fig. 3 and main text). Migration to American Samoa (AS)
traces

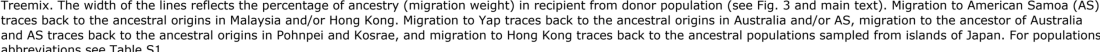

b

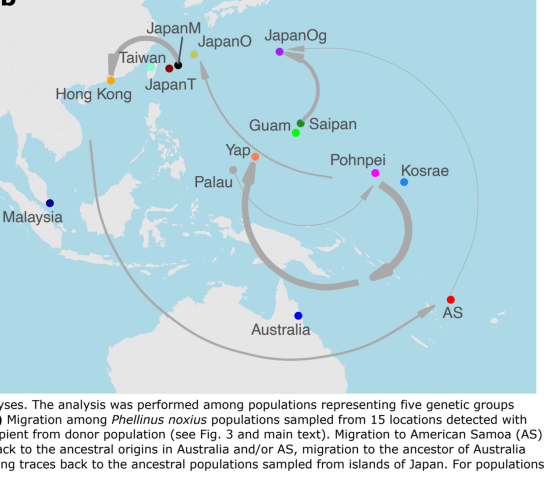

